\title{
ON EXTENDING FREE GROUP ACTIONS ON SPHERES AND A CONJECTURE OF IWASAWA \\ BY
}

FRANK CONNOLLY AND ROBERT GEIST

\begin{abstract}
A transfer map for Reidemeister torsion is defined and used to determine whether free actions of $\mathbf{Z} / k$ on $S^{2 n+1}, n>1$, extend to free actions of $\mathbf{Z} / h k$. It is shown that for $k$ odd, every free $\mathbf{Z} / k$ action on $S^{2 n+1}, n>1$, extends to a free $\mathbf{Z} / 2 k$ action. For prime $p$, extension of an arbitrary free $\mathbf{Z} / p$ action to a free $\mathbf{Z} / p^{2}$ action is reduced to a long-standing conjecture of Iwasawa.
\end{abstract}

0. Introduction and statement of results. In this paper we address the following basic question: Let $\pi$ be a finite group, $\rho$ a subgroup. When does a.free action of $\rho$ on $S^{2 n+1}$ extend to a free action of $\pi$ ?

Some of our results apply in general, but we concentrate on the case where $\pi$ is a cyclic group. In this case we answer the above question completely, reducing the issue to algebraic number theory. If $\rho=1$, our results can be viewed as an addendum to [1] which solved this question for $\rho=1$ and $\pi$ an odd order cyclic group. We will now briefly summarize our results.

We write an action of $\pi$ as a map $\mu: \pi \times S^{2 n+1} \rightarrow S^{2 n+1}$ and we say $\mu_{1}, \mu_{2}$ are equivalent if there is a homeomorphism $f: S^{2 n+1} \rightarrow S^{2 n+1}$ such that $f \mu_{1}(T, x)=$ $\mu_{2}(T, f(x))$ for all $(T, x) \in \pi \times S^{2 n+1}$. For $\pi$ cyclic of order $h$, the prototype is of course the linear action of $\mathbf{Z} / h$ on $S^{2 n+1} \subset \mathbf{C}^{n+1}$ given by $\mu_{0}\left(T,\left(z_{0}, z_{1}, \ldots, z_{n}\right)\right)=$ $\exp (2 \pi i / h)\left(z_{0}, z_{1}, \ldots, z_{n}\right)$. We write $\Delta(\mu)$ for the Reidemeister torsion of the action $\mu: \pi \times S^{2 n+1} \rightarrow S^{2 n+1}$, following Milnor [4]. $\Delta(\mu)$ is a unit in the ring $\mathbf{Q} R_{\pi}$, defined by $\mathbf{Q} \pi /(\Sigma)$, where $\Sigma$ denotes the sum of the elements of $\pi$. It is a basic invariant of the action. For the standard linear action mentioned above, $\Delta\left(\mu_{0}\right)=(T-1)^{n+1}$ (see $[4$, p. 406]). Actually it is more convenient to keep tabs on the quotient

$$
\Delta(\mu) / \Delta\left(\mu_{0}\right)=\Delta(\mu) \cdot(T-1)^{-n-1} \in \mathbf{Q} R_{\pi}^{\times} /( \pm \pi) .
$$

In fact, let $R_{\pi}=\mathbf{Z} \pi /(\Sigma)$ with $j:\left(R_{\pi}\right)^{\times} \rightarrow \mathbf{Q} R_{\pi}^{\times}$the natural inclusion map. Then Wall has shown [9, Theorem 14.E.3] that

0.1. Lemma. $\Delta(\mu) / \Delta\left(\mu_{0}\right)=j(u)$ for some unique unit $u \in\left(R_{\pi}\right)^{\times} /( \pm \pi)$. Moreover, given a unit $u \in\left(R_{\pi}\right)^{\times}$there is a cellular free action $\mu$ on a finite complex homotopically equivalent to $S^{2 n+1}$ such that $\Delta(\mu) / \Delta\left(\mu_{0}\right)=j(u) \bmod ( \pm \pi)$.

For this reason, this unit $u \in\left(R_{\pi}\right)^{\times} /( \pm \pi)$ is called the associated unit of $\mu$.

Received by the editors April 14, 1980 and, in revised form, June 24, 1981.

1980 Mathematics Subject Classification. Primary 57S17, 57S25, 57Q10; Secondary 18F25, 12A35. 
After a surgery calculation-carried out in Part I-we define, for each finite group $\pi$ and subgroup $\rho$, a transfer map,

$$
\begin{aligned}
& \operatorname{tr}: K_{1}\left(R_{\pi}\right) /( \pm \pi) \rightarrow K_{1}\left(R_{\rho}\right) /( \pm \rho) \text { and } \\
& \operatorname{tr}: K_{1}\left(\mathbf{Q} R_{\pi}\right) /( \pm \pi) \rightarrow K_{1}\left(\mathbf{Q} R_{\rho}\right) /( \pm \rho)
\end{aligned}
$$

and we prove that $\operatorname{tr} \Delta(\mu)=\Delta(\bar{\mu})$ whenever $\mu: \pi \times X \rightarrow X$ is a free action for which the torsion is defined, and $\bar{\mu}$ is its restriction to $\rho$. This is Theorem 1.7 , and we feel it should be of independent interest.

Now it is known that

LEMMA. $K_{1}\left(R_{\pi}\right)=R_{\pi}^{\times}$if $\pi$ is a finite cyclic group.

(For a proof, see the Remark following 1.6.B.)

Hence, if $\pi$ is a cyclic group our definition of the transfer provides a homomorphism

$$
\operatorname{tr}:\left(R_{\pi}\right)^{\times} /( \pm \pi) \rightarrow\left(R_{\rho}\right)^{\times} /( \pm \rho)
$$

We then prove

THEOREM 1. Let $\bar{\mu}$ be a free action of $\rho=\mathbf{Z} / k$ on $S^{2 n+1}, n \geqslant 2$. Let $\bar{u} \in$ $\left(R_{\rho}\right)^{\times} /( \pm \rho)$ be its associated unit. Then $\bar{\mu}$ extends to a free action of $\mathbf{Z} / h k=\pi$ if and only if $\bar{u}$ is in the image of $\operatorname{tr}:\left(R_{\pi}\right)^{\times} /( \pm \pi) \rightarrow\left(R_{\rho}\right)^{\times} /( \pm \rho)$.

If $p$ is a prime number it then turns out that the problem of extending a $\mathbf{Z} / p^{r}$ action to a $\mathbf{Z} / p^{r+1}$ action is closely related to an old conjecture of Iwasawa in [2]. This conjecture states that the norm map $N: \mathbf{Z}\left(\zeta_{r+1}\right)^{\times} \rightarrow \mathbf{Z}\left(\zeta_{r}\right)^{\times}$is always onto, where $\zeta_{r}$ denotes a primative $p^{r}$ th root of unity. Iwasawa's conjecture would follow at once from Vandiver's conjecture (which says that $p$ does not divide the second factor of the ideal class group of $\left.\mathbf{Z}\left(\zeta_{1}\right)\right)$. Vandiver's conjecture is known to be true for all primes $<125,000$. (Cf. [2, p. 556 and 12].)

The relationship of this to group actions is as follows: If $\pi=\mathbf{Z} / p^{r+1}, \rho=\mathbf{Z} / p^{r}$, define a map $\varepsilon: R_{\pi} \rightarrow \mathbf{Z}\left(\zeta_{r}\right)$ by sending $T$ to $\zeta_{r}$. $\varepsilon$ induces $\varepsilon_{*}:\left(R_{\rho}\right)^{\times} \rightarrow \mathbf{Z}\left(\zeta_{r}\right)^{\times}$ sending $( \pm \rho)$ to $C$, the group of roots of unity.

THEOREM 2. Let $\bar{\mu}$ be a free action of $\mathbf{Z} / p^{r}$ on $S^{2 n+1}, n \geqslant 2$. Let $\bar{u}$ be its associated unit.

(A) If $\varepsilon_{*}(\bar{u})$ is not in the image of $N: \mathbf{Z}\left(\zeta_{r+1}\right)^{\times} \rightarrow \mathbf{Z}\left(\zeta_{r}\right)^{\times} / C$, then $\bar{\mu}$ does not extend to a $\mathbf{Z} / p^{r+1}$ action.

(B) Suppose $r=1$, and suppose Iwasawa's conjecture holds for $p$. Then $\bar{\mu}$ extends to a free $\mathbf{Z} / p^{2}$ action on $S^{2 n+1}$.

REMARK. Despite the explicit nature of Theorem 1, we can provide no example of a free $\mathbf{Z} / k$ action which fails to extend to a free $\mathbf{Z} / h k$ action. John Ewing has shown [11], using our results, that for each $p$, there is a $\mathbf{Z} / p^{r}$ action which does not extend to a $\mathbf{Z} / p^{r+1}$ action.

THEOREM 3. Let $k$ be an odd integer. Every free $\mathbf{Z} / k$ action on $S^{2 n+1}, n>1$, extends to a free $\mathbf{Z} / 2 k$ action. 
Here is an outline of the rest of the paper.

Part I of $\S 1$ is a surgery theoretic calculation showing that the natural transfer map of the two surgery exact sequences involved is surjective. Part II constructs the transfer map needed to study the Reidemeister torsion; we then use this to prove Theorem 1. $\$ 2$ gives the proof of Theorems 2 and 3.

We would like to give a word of thanks here to John Ewing and to John Cruthirds for a number of very useful conversations.

\section{The basic condition for extending actions.}

PART I. Our goal in this part of $\S 1$ is to establish

Proposition 1.1. Let $L^{2 n+1}$ be a finite Poincaré complex with $\pi_{1}(L)=\mathbf{Z} /$ hk and universal cover $\simeq S^{2 n+1}$. Let $\bar{L}$ denote its $h$-fold cover. Then the transfer map,

$$
\pi^{*}: S_{\text {Top }}^{s}(L) \rightarrow S_{\text {Top }}^{s}(\bar{L}),
$$

is onto.

(As in Wall [9], $S_{\text {Top }}^{\text {s }}(L)$ denotes the Top-structures on $L$.)

This will be done analyzing the following map of surgery exact sequences:

$$
\begin{array}{ccccccc}
\tilde{L}_{2 n+2}^{s}(\mathbf{Z} / k) & \rightarrow & S_{\text {Top }}^{s}(\bar{L}) & \rightarrow & N M(\bar{L}) & \stackrel{\bar{\sigma}}{\rightarrow} & L_{2 n+1}^{s}(\mathbf{Z} / k) \\
\uparrow \operatorname{tr} & & \uparrow \pi^{*} & & \uparrow \pi^{*} & & \uparrow \operatorname{tr} \\
\tilde{L}_{2 n+2}^{s}(\mathbf{Z} / h k) & \rightarrow & S_{\text {Top }}^{s}(L) & \rightarrow & N M(L) & \stackrel{\sigma}{\rightarrow} & L_{2 n+1}^{s}(\mathbf{Z} / h k)
\end{array}
$$

Here $\tilde{L}_{n}^{s}(G)=$ the cokernel of $L_{n}^{s}(e) \rightarrow L_{n}^{s}(G)$, and $N M$ denotes the set of bordism classes of Top-normal maps.

It is well known that such an $L$ is homotopically equivalent to some standard lens space $L\left(h k, \theta_{0}, \theta, \ldots, \theta_{n}\right)$ where $\theta_{i}$ are integers prime to $h k$. Choose such a homotopy equivalence: it yields an element of $N M(L)$ and its cover is an element of $N M(\bar{L})$. This choice then allows us to identify $N M(L)$ with $\left[L, G /\right.$ Top] (see [6 or 9]) and $\pi^{*}$ with the group homomorphism, $\pi^{*}:[L, G /$ Top $] \rightarrow[\bar{L}, G /$ Top $]$. Our first step is

LEMma 1.3. $\pi^{*}:[L, G /$ Top $] \rightarrow[\bar{L}, G /$ Top $]$ is an epimorphism. Its kernel is a finite group of order $h^{[n / 2]}$ unless $k$ is odd and $h$ is even. In this case its order is $2^{[(n+1) / 2]} h^{[n / 2]}$.

Proof. $[L, G /$ Top $]=\left[L, G / \operatorname{Top}_{(2)}\right] \oplus\left[L, G /\right.$ Top $\left._{(\text {odd })}\right]$ (since both localizations are finite) and this is isomorpic to $\tilde{H}^{4 *}\left(L ; \mathbf{Z}_{(2)}\right) \oplus H^{4 *+2}(L ; \mathbf{Z} / 2) \oplus \tilde{K} O^{\circ}(L)_{\text {(odd) }}$. Similarly for $\bar{L}$. So the lemma is an immediate consequence of the following three statements. We first write $h$ as $h=h_{2} \cdot h_{\text {odd }}$ where $h_{2}$ is a power of 2 and $h_{\text {odd }}$ is odd.

(i) $\pi^{*}: \tilde{H}^{4 *}\left(L ; \mathbf{Z}_{(2)}\right) \rightarrow \tilde{H}^{4 *}\left(\bar{L} ; \mathbf{Z}_{(2)}\right)$ is surjective; its kernel has order $h_{2}^{[n / 2]}$.

(ii) $\pi^{*}: H^{4^{*}+2}(L ; \mathbf{Z} / 2) \rightarrow H^{4 *+2}(\bar{L} ; \mathbf{Z} / 2)$ is surjective; its kernel has order $2^{[n+1 / 2]}$ if $k$ is odd and $h$ is even, and otherwise it is injective.

(iii) $\pi^{*}: \tilde{K} O(L) \rightarrow \tilde{K} O(\bar{L})$ is onto; its kernel has order $\left(h_{\text {odd }}\right)^{[n / 2]}$. 
Proof OF (i) AND (ii). It is an easy exercise (using the fibration $S^{2 n+1} \rightarrow \bar{L} \rightarrow$ $K(\mathbf{Z} / k, 1))$ to prove, for $A=\mathbf{Z}_{(2)}$ or $\mathbf{Z} / 2$, that $H^{2 *}(\bar{L} ; A)=(A / k A)[\bar{x}] /(\bar{x})^{n+1}$ where $\operatorname{deg} \bar{x}=2$, and similarly for $L$. The surjectivity of $\pi^{*}: H^{2}(L ; A) \rightarrow H^{2}(\bar{L} ; A)$ is trivial from the universal coefficient theorem and the behavior of $\pi_{*}$ on the fundamental groups. From this it is clear that $\pi^{*}$ is onto with kernel as specified in (i) and (ii).

To prove (iii) let $C$ be the mapping cone of $\pi$. Proceeding as above, one easily computes: $H^{i}\left(C ; \mathbf{Z}_{\text {(odd) }}\right)=\mathbf{Z} / h_{\text {odd }}$ if $i$ is even and $0<i \leqslant 2 n+2$, and $H^{i}\left(C ; \mathbf{Z}_{\text {(odd) }}\right)$ $=0$ otherwise. Hence the Atiyah-Hirzebruch spectral sequence for $K O^{*}(C)_{\text {(odd) }}$ collapses $\left(E_{2}^{p q}=0\right.$ if $p$ or $q$ is odd) and we get $\tilde{K} O^{1}(C)_{(\text {odd })}=0, \tilde{K} O^{\circ}(C)_{(\text {odd })}=$ a group of order $\left(h_{\text {odd }}\right)^{[(n+1) / 2]}\left(E_{2}^{4 i,-4 i}=\mathbf{Z} /\left(h_{\text {odd }}\right), 0<i \leqslant[(n+1) / 2]\right)$. so we get an exact sequence

$$
\begin{aligned}
0 & \rightarrow \tilde{K} O^{-1}(L)_{(\text {odd })} \stackrel{\pi^{*}}{\rightarrow} \tilde{K} O^{-1}(\bar{L})_{(\text {odd })} \rightarrow \tilde{K} O^{\circ}(C)_{(\text {odd })} \\
& \rightarrow \tilde{K} O^{\circ}(L)_{(\text {odd }) ~} \stackrel{\pi^{*}}{\rightarrow} \tilde{K} O^{\circ}(\bar{L})_{(\text {odd })} \rightarrow 0
\end{aligned}
$$

However, $\tilde{K} O^{-1}(\bar{L})_{(\text {odd) }}=0$ if $n$ is even (use the Atiyah-Hirzebruch spectral sequence), and for $n$ odd $\tilde{K} O^{-1}(\bar{L})=\mathbf{Z}_{\text {(odd) }}$ with $\operatorname{Im}\left(\pi^{*}\right)$ of index $\left(h_{\text {odd }}\right)$. So for any $n$, $\pi^{*}$ on the right is an epimorphism with kernel of order $\left(h_{\text {odd }}\right)^{[n / 2]}$. This proves Lemma 1.3.

Now we return to diagram (1.2) and glue together some ideas of Petrie and Wall to observe:

LEMMA 1.4. The map

$$
\operatorname{tr}: \tilde{L}_{2 n+2}^{s}(\mathbf{Z} / h k) \rightarrow \tilde{L}_{2 n+2}^{s}(\mathbf{Z} / k)
$$

is surjective. Also $\tilde{L}_{2 n+2}^{s}(\mathbf{Z} / k)$ acts freely on $S_{\text {Top }}^{s}(\bar{L})$ and similarly for $\mathbf{Z} / h k$ and $L$.

Proof. Following Wall [8] we write $\chi_{k}: L_{2 n+2}^{s}(\mathbf{Z} / k) \rightarrow \mathbf{C}(\mathbf{Z} / k)$ for the signature map, where $\mathbf{C}(\pi)$ denotes the representation ring of $\pi$ (over $\mathbf{C}$ ). According to [8], $\operatorname{Im} \chi_{k}=\left\{4\left(x+(-1)^{n+1} \bar{x}\right) \mid x \in \mathbf{C}(\mathbf{Z} / k)\right\}$. Similarly for $\chi_{h k}$. The diagram

$$
\begin{array}{ccc}
L_{2 n+2}^{s}(\mathbf{Z} / k) & \stackrel{\chi_{k}}{\rightarrow} & \mathbf{C}(\mathbf{Z} / k) \\
\uparrow \operatorname{tr} & & \uparrow p \\
L_{2 n+2}^{s}(\mathbf{Z} / h k) & \stackrel{\chi_{h k}}{\rightarrow} & \mathbf{C}(\mathbf{Z} / h k)
\end{array}
$$

commutes where $p$ denotes restriction. Since each representation of $\mathbf{Z} / k$ is the restriction of a $\mathbf{Z} / h k$ representation it is clear that $p \operatorname{Im} \chi_{h k}=\operatorname{Im} \chi_{k}$.

But according to Wall [8], $\chi$ is injective for $n$ odd and $\operatorname{Ker} \chi=L_{2 n+2}(e)$ for $n$ even for these groups. It follows at once that tr is a surjection on $\tilde{L}_{2 n+2}^{s}(\mathbf{Z} / k)$.

Now $\tilde{L}_{2 n+2}^{s}(\mathbf{Z} / k)$ is a free abelian, and Petrie [5,2.3 and 2.10] shows that a subgroup of $\tilde{L}_{2 n+2}^{s}(\mathbf{Z} / k)$ acts freely on $S_{\text {Top }}^{s}(\bar{L})$. But the rank of this subroup equals the rank of $\tilde{L}_{2 n+2}^{s}(\mathbf{Z} / k)$ (as computed by Wall [7]). It follows then that $\tilde{L}_{2 n+2}^{s}(\mathbf{Z} / k)$ itself is acting freely. This completes the proof. 
The final step in this section concerns the right side of (1.2), where we now look at the kernel of $\sigma: N M(L) \rightarrow L_{2 n+1}^{s}\left(\pi_{1}(L)\right)$. Recall $N M(L)$ is a group via its identification with $[L, G /$ Top].

LEMMA 1.5. The map $\sigma$ is a homomorphism and the restriction of $\pi^{*}$ to $\pi^{*}$ : $\operatorname{Ker} \boldsymbol{\sigma} \rightarrow \operatorname{Ker} \overline{\boldsymbol{\sigma}}$ is an epimorphism.

Proof. $L_{2 n+1}^{s}(\mathbf{Z} / h k)=0$ unless $n$ is odd and $h k$ even, and in this case $L_{2 n+1}^{s}(\mathbf{Z} / h k)=\mathbf{Z} / 2$, with the surgery obstruction given by

$$
\sigma(f)=\left\langle a_{1}\left(1+a_{2}^{2}\right)^{r} f^{*} k,[L]\right\rangle
$$

(see [9, p. 210]). Here $f \in\left[L, G /\right.$ Top], $2 r=n+1, a_{i} \in H^{i}(L ; \mathbf{Z} / 2)$ is the nonzero element, and $k \in H^{4^{*}+2}(G / \mathrm{Top} ; \mathbf{Z} / 2)$ is a certain primitive class. The primitivity of $k$ yields immediately that $\sigma$ is a homomorphism. To prove $\pi^{*} \operatorname{Ker} \sigma=\operatorname{Ker} \bar{\sigma}$ we can assume, using 1.3, that $n$ is odd and $h k$ is even.

Case 1. $k$ even. Then if $\bar{f} \in \operatorname{Ker} \bar{\sigma}$ and $f$ is an element of $[L, G /$ Top] such that $\pi^{*} f=\bar{f}$ we see

$$
\begin{aligned}
\sigma(f) & =\left\langle a_{1}\left(1+a_{2}^{2}\right)^{r} f^{*} k,[L]\right\rangle \\
& =\left\langle\left(\operatorname{tr} \bar{a}_{1}\right)\left(1+a_{2}^{2}\right)^{r} f^{*} k,[L]\right\rangle \quad \text { since } \operatorname{tr} \bar{a}_{1}=a_{1} \\
& =\left\langle\operatorname{tr}\left\{\bar{a}_{1} \cdot \pi^{*}\left(\left(1+a_{2}^{2}\right)^{r} f^{*} k\right)\right\},[L]\right\rangle \quad \text { since }(\operatorname{tr} x) y=\operatorname{tr}\left(x \cdot \pi^{*} y\right) \\
& =\left\langle\operatorname{tr}\left\{\bar{a}_{1} \cdot\left(1+\bar{a}_{2}^{2}\right)^{r} \bar{f}^{*} k\right\},[L]\right\rangle \quad \text { since } \pi^{*} a_{2}=\bar{a}_{2} \\
& =\left\langle\bar{a}_{1}\left(1+\bar{a}_{2}^{2}\right)^{r} \bar{f}^{*} k,[\bar{L}]\right\rangle \quad \text { since } \operatorname{tr}[L]=[\bar{L}] \\
& =\sigma(\bar{f})=0 .
\end{aligned}
$$

Hence $f \in \operatorname{Ker} \sigma$ and $\pi^{*}: \operatorname{Ker} \sigma \rightarrow \operatorname{Ker} \bar{\sigma}$ is onto in this case.

Case 2. $k$ odd. Then $[\bar{L}, G /$ Top $]=\operatorname{Ker} \bar{\sigma}$ is an odd order group (as seen, for example, in the proof of 1.3 ) and the odd primary summand of $[L, G /$ Top] must map onto it by Lemma 1.3. But this summand is in Ker $\sigma$ since $\sigma$ is a homomorphism. This completes the proof.

Proof of Proposition 1.1. Using 1.3, 1.4 and 1.5 we see that diagram 1.2 reduces to:

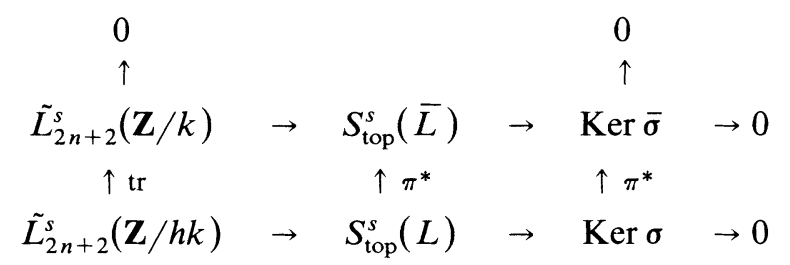

Hence 1.1 becomes a simple diagram chase, and we are done.

PART II. We wish to make a statement concerning the behavior of the Reidmeister torsion under a transfer map so we will now define this transfer map.

1.6. Construction. Let $A$ be a commutative ring (in practice $A=\mathbf{Q}$ or $\mathbf{Z}$ ). Set $A R_{\pi}=A(\pi) /(\Sigma), A R_{\rho}=A(\rho) /(\bar{\Sigma})$, where $\rho$ is a subgroup of the finite group $\pi$, 
and $\bar{\Sigma}$ is the sum of the elements of $\rho$. The multiplicative group $\pm \pi$ in $(A \pi)^{\times}$ determines a subgroup of $K_{1}\left(A R_{\pi}\right)$ denoted $( \pm \pi)$, the homomorphic image of $\pm \pi$. We construct here a homomorphism

$$
\operatorname{tr}: K_{1}\left(A R_{\pi}\right) /( \pm \pi) \rightarrow K_{1}\left(A R_{\rho}\right) /( \pm \rho) .
$$

$A(\pi)$ is a free left $A(\rho)$ module with base $g_{1}, \ldots, g_{h}$, a complete set of right coset representatives of $\rho$ in $\pi$. Let $T=\bar{\Sigma} \cdot A(\pi)$, a right ideal. $A(\pi) / T$ is a left $A(\rho)$ module with $\bar{\Sigma} \cdot(A(\pi) / t)=0$ so that $A(\pi) / T$ becomes a left $A R_{\rho}$ module. In fact, $A(\pi) / T$ is a free left $A R_{\rho}$ module with base $g_{1} \bmod T \cdots g_{h} \bmod T$. This base defines an isomorphism $A(\pi) / T \approx\left(A R_{\rho}\right)^{h}$ of left $A R_{\rho}$ modules, and an induced isomorphism from $\operatorname{End}(A(\pi) / T)$, the ring of $A R_{\rho}$ endomorphisms of $A(\pi) / T$, to the matrix ring $M\left(h, A R_{\rho}\right)$. Denote this

$$
\lambda: \operatorname{End}(A(\pi) / T) \approx M\left(h, A R_{\rho}\right) .
$$

Now right multiplication by elements of $A(\pi)$ defines a map $A(\pi) \rightarrow \operatorname{End}(A(\pi) / T)$ sending $\Sigma$ to 0 . This induces a ring homomorphism $r: A R_{\pi} \rightarrow \operatorname{End}(A(\pi) / T)$.

So the ring homomorphism $\lambda r: A R_{\pi} \rightarrow M\left(h, A R_{\rho}\right)$ yields a ring homomorphism $(\lambda r)_{*}: M\left(m, A R_{\pi}\right) \rightarrow M\left(m h, A R_{\rho}\right)$ given by $(\lambda r)_{*}\left(a_{i j}\right)=\left(\lambda r\left(a_{i j}\right)\right)$ (a matrix of blocks). The induced homomorphism on the $K_{1}$ level is denoted

$$
\operatorname{tr}: K_{1}\left(A R_{\pi}\right) \rightarrow K_{1}\left(A R_{\rho}\right) .
$$

We claim that $\operatorname{tr}( \pm \pi) \subset( \pm \rho)$. For, if $x \in \pi$, then for each coset representative $g_{i}$ we have $g_{i} x=\bar{x}(i) \cdot g_{j(x, i)}$ for some element $\bar{x}(i) \in \rho$ and some integer $j(x, i)$ between 1 and $h$. It follows that $\lambda r( \pm x)= \pm P \cdot D(\bar{x}(1), \ldots, \bar{x}(h))$ where $P$ is a permutation matrix and $D$ denotes a diagonal matrix. Hence, $\operatorname{tr}( \pm x)= \pm \bar{x}(1) \cdot$ $\bar{x}(2) \cdots \bar{x}(h) \in( \pm \rho)$.

This leaves us with an induced map

$$
\operatorname{tr}: K_{1}\left(A R_{\pi}\right) /( \pm \pi) \rightarrow K_{1}\left(A R_{\rho}\right) /( \pm \rho) .
$$

The elementary properties below are easily checked.

1.6.A. If $A \stackrel{f}{\rightarrow} A^{\prime}$ is a ring homomorphism, the following diagram commutes:

$$
\begin{array}{ccc}
K_{1}\left(A R_{\pi}\right) /( \pm \pi) & \stackrel{\operatorname{tr}}{\rightarrow} & K_{1}\left(A R_{\rho}\right) /( \pm \rho) \\
\downarrow f^{*} & & \downarrow f^{*} \\
K_{1}\left(A^{\prime} R_{\pi}\right) /( \pm \pi) & \stackrel{\operatorname{tr}}{\rightarrow} & K_{1}\left(A^{\prime} R_{\rho}\right) /( \pm \rho)
\end{array}
$$

1.6.B. Suppose given a ring epimorphism $\varphi: A(\pi) \rightarrow \Lambda$ with $\bar{\Lambda}=\varphi(A(\rho))$ and with $\varphi(\bar{\Sigma})=0$. Suppose $\Lambda$ is free over $\bar{\Lambda}$ with base $\varphi\left(g_{1}\right), \ldots, \varphi\left(g_{h}\right)$. Then there is a commutative square:

$$
\begin{array}{ccc}
K_{1}\left(A R_{\pi}\right) & \stackrel{\varphi_{*}}{\rightarrow} & K_{1}(\Lambda) \\
\downarrow \operatorname{tr} & & \downarrow N \\
K_{1}\left(A R_{\rho}\right) & \stackrel{\bar{\varphi}_{*}}{\rightarrow} & K_{1}(\bar{\Lambda})
\end{array}
$$


Here $\bar{\varphi}: A R_{\rho} \rightarrow \bar{\Lambda}$ is the epimorphism induced by $\varphi$, and $N$ is the "norm" map induced by the isomorphism of rings, $M(n, \Lambda) \rightarrow M(n h, \bar{\Lambda})$ which this basis of $\Lambda$ over $\bar{\Lambda}$ defines.

REMARK. If $\pi$ is a finite cyclic group, $S K_{1}(\mathbf{Z} \pi)=\{1\}$ (see [10, p. 623]). Hence, $K_{1}(\mathbf{Z} \pi)=\mathbf{Z} \pi^{\times}$and one sees at once that $K_{1}\left(R_{\pi}\right)=R_{\pi}^{\times}$, by examining the exact sequence of the Cartesian square:

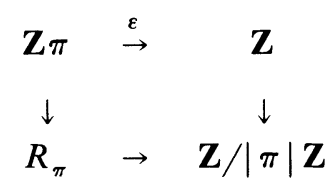

Also $K_{1}\left(\mathbf{Q} R_{\pi}\right)=\left(\mathbf{Q} R_{\pi}\right)^{\times}$because $\mathbf{Q} R_{\pi}$ is a product of fields.

Now we show how the torsion of a complex behaves under this transfer map.

So let $L$ denote a finite $\mathrm{CW}$ complex with fundamental group $\pi$, a finite group, universal cover $\tilde{L}$ and intermediate cover $\bar{L}$ corresponding to a subgroup $\rho$ of $\pi$. We assume $\pi$ acts trivially on $H_{*}(\tilde{L} ; \mathbf{Q})$. In this case, Milnor [4, p. 405], defines its Reidemeister torsion $\Delta(L)$ in $K_{1}\left(\mathbf{Q} R_{\pi}\right) /( \pm \pi)$. We shall prove

THEOREM 1.7. $\Delta(\bar{L})=\operatorname{tr} \Delta(L)$.

Proof. Recall from [4] how $\Delta$ is defined. If $C(\tilde{L})$ denotes the cellular chain complex then $C(\tilde{L}) \otimes_{\mathbf{Z}_{\pi}} \mathbf{Q} R_{\pi}$ (hereafter denoted $C_{\pi}$ ) is acyclic and based, over $\mathbf{Q} R_{\pi}$. Similarly for $C(\tilde{L}) \otimes_{\mathbf{z}_{\rho}} \mathbf{Q} R_{\rho}$ (written $C_{\rho}$ ), over $\mathbf{Q} R_{\rho} . \Delta(L)=\Pi_{i}\left[c_{i} / c_{i}^{\prime}\right]$ where $c_{i}$ denotes the basis of cells for $\left(C_{\pi}\right)_{i}$ and $c_{i}^{\prime}$ denotes the basis determined by the acyclicity of $C_{\pi}$, and $\left[c_{i} / c_{i}^{\prime}\right]$ denotes the class of the change of basis matrix $\left(c_{i} / c_{i}^{\prime}\right)$ in $G L\left(m_{i}, \mathbf{Q} R_{\pi}\right)\left(m_{i}=\operatorname{rank} C_{i}\right.$, over $\left.\mathbf{Z} \pi\right)$.

Now if $g_{1}, \ldots, g_{h}$ are a complete set of coset representatives of $\rho$ in $\pi$, each basis $c_{i}$ for $\left(C_{\pi}\right)_{i}$ determines a basis for $\left(C_{\rho}\right)_{i}$ over $\mathbf{Q} R_{\rho}$ - namely: $c_{i} g_{1} \cup c_{i} g_{2} \cup \cdots \cup c_{i} g_{h}$ which we write $\bar{c}_{i}$. Observe that $\bar{c}_{i}^{\prime}, i=0,1,2, \ldots$, are the bases for $\left(C_{\rho}\right)_{i}$ determined by its acyclicity. Going back to 1.6 , we check that $(\lambda r)_{*}\left(c_{i} / c_{i}^{\prime}\right)=\left(\bar{c}_{i} / \bar{c}_{i}^{\prime}\right)$ in $G L\left(m_{i} h, \mathbf{Q} R_{\rho}\right)$. Hence $\operatorname{tr}(L)=\prod_{i} \operatorname{tr}\left[c_{i} / c_{i}^{\prime}\right]=\prod_{i} \lambda r_{*}\left(c_{i} / c_{i}^{\prime}\right)=\prod_{i}\left[\bar{c}_{i} / \bar{c}_{i}^{\prime}\right]=\Delta(\bar{L})$ because, by its definition, it is clear that $\bar{c}_{i}$ is the basis of cells for $\left(C_{\rho}\right)_{i}$ over $\mathbf{Q} R_{\rho}$. This completes the proof.

1.8. Proof of Theorem 1. First we show that if $\bar{u}$ is the associated unit of an action $\bar{\mu}: \mathbf{Z} / k \times S^{2 n+1} \rightarrow S^{2 n+1}$ which does extend to a free $\mathbf{Z} / h k$ action $\mu$, we have $\bar{u}=\operatorname{tr} u$ for some $u \in\left(R_{\pi}\right)^{\times} /( \pm \pi)$. In fact $u$ can be taken to be the associated unit of $\mu$. To see this we calculate

$$
\Delta(\mu)=(T-1)^{n+1} j(u) \text { and } \Delta(\bar{\mu})=\left(T^{h}-1\right)^{n+1} j(\bar{u})
$$

by 0.1 . By Theorem 1.7, $\operatorname{tr} \Delta(\mu)=\Delta(\bar{\mu})$ and $\operatorname{tr}(T-1)^{n+1}=\left(T^{h}-1\right)^{n+1}$ (since $\left.\Delta\left(\mu_{0}\right)=(T-1)^{n+1}\right)$. Hence we see $j \bar{u}=\operatorname{tr} j(u)=j \operatorname{tr} u$ by 1.6.A. But

$$
j: R_{\rho}^{\times} /( \pm \rho) \rightarrow \mathbf{Q} R_{\rho}^{\times} /( \pm \rho)
$$

is evidently injective. So $\operatorname{tr} u=\bar{u}$ as required.

Conversely, now we suppose $\bar{\mu}$ is an action with associated unit $\bar{u}$ and that $\bar{u}=\operatorname{tr} u$ for some unit in $\left(R_{\pi}\right)^{\times} /( \pm \pi)$. By 0.1 , there is a finite complex $L$ with fundamental 
group $\pi$, universal cover $\simeq S^{2 n+1}$, such that $\Delta(L)=(T-1)^{n+1} j(u)$. Let $\bar{L}$ be the $h$-fold cover of $L$. By Theorem 1.7, $\Delta(\bar{L})=\left(T^{h}-1\right)^{n+1} j(\bar{u})=\Delta(\bar{M})$, where $\bar{M}$ is the orbit manifold of the action $\bar{\mu}: \rho \times S^{2 n+1} \rightarrow S^{2 n+1}$. But this implies that $\bar{M}$ is simple homotopy equivalent to $\bar{L}$ via a map $\bar{f}: \bar{M} \rightarrow \bar{L}$ say. Hence $(\bar{M}, \bar{f}) \in S_{\text {Top }}^{s}(\bar{L})$. But by Proposition 1.1, there is an $(M, f)$ in $S_{\text {Top }}^{s}(L)$ whose cover is $(\bar{M}, \bar{f})$. This means that $M$ is the orbit space of a free action of $\pi$ on $\tilde{M}$. Its restriction to an action of $\rho$ is just $\bar{\mu}$. Thus $\bar{\mu}$ extends as required. This completes the proof of Theorem 1.

2. Proof of Theorem 2. We now turn to free actions of cyclic groups of order $p^{r}$ with the aim of proving Theorem 2.

Proof of Theorem 2(A). By Theorem $1, \bar{\mu}$ extends to a free action $\mu$ only if $\bar{u}=\operatorname{tr} u$ for some unit $u$ in $\left(R_{\pi}\right)^{\times}$. But by 1.6.B the diagram below commutes. So $\bar{\mu}$ does not extend unless $\varepsilon_{*}(\bar{u})$ is in the image of $N$.

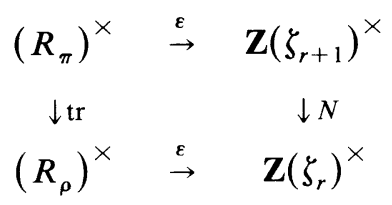

Proof of Theorem 2(B). If $\pi=\mathbf{Z} / p^{2}$, and $\zeta_{i}=$ the primitive $p^{i}$ th root of unity, we have the exact sequence,

$$
1 \rightarrow R_{\pi}^{\times} \stackrel{\lambda_{1} \oplus \lambda_{2}}{\rightarrow} Z\left(\zeta_{1}\right)^{\times} \oplus Z\left(\zeta_{2}\right)^{\times} \stackrel{k}{\rightarrow}\left(Z\left(\zeta_{1}\right) /(p)\right)^{\times},
$$

$\lambda_{i} f(T)=f\left(\zeta_{i}\right), \quad k\left(f_{1}\left(\zeta_{1}\right), f_{2}\left(\zeta_{2}\right)\right)=f_{1}\left(\zeta_{1}\right)^{-1} f_{2}\left(\zeta_{1}\right)$ mod $p \quad$ (obtained by noting $\left.\mathbf{Z}[T] / \Phi_{1} \cdot \Phi_{2}=R_{\pi}, \mathbf{Z}[T] /\left(\Phi_{1}, \Phi_{2}\right)=\mathbf{Z}\left(\zeta_{1}\right) /(p)\right)$.

Now $R_{\rho}=\mathbf{Z}\left(\zeta_{1}\right)$ and it is easy to see from the definition of $\operatorname{tr}$ that $\operatorname{tr}=N \circ \lambda_{2}$ : $R_{\pi}^{\times} \rightarrow \mathbf{Z}\left(\zeta_{2}\right)^{\times} \rightarrow \mathbf{Z}\left(\zeta_{1}\right)^{\times}$where $N$ is a norm map as in 1.6.B. Iwasawa's conjecture asserts that $N: \mathbf{Z}\left(\zeta_{2}\right)^{\times} \rightarrow \mathbf{Z}\left(\zeta_{1}\right)^{\times}$is surjective. So if Iwasawa's conjecture holds, we need only prove that $\operatorname{tr}$ is onto (by Theorem 1). So let $\bar{u} \in \mathbf{Z}\left(\zeta_{1}\right)^{\times}$. We are given that $\bar{u}=N\left(f\left(\zeta_{2}\right)\right)$ for some unit $f\left(\zeta_{2}\right)$ in $\mathbf{Z}\left(\zeta_{2}\right)^{\times}$. Consider $z=\left(\bar{u}, f\left(\zeta_{2}\right)\right) \in \mathbf{Z}\left(\zeta_{1}\right)^{\times}$ $\oplus \mathbf{Z}\left(\zeta_{2}\right)^{\times}$. It is well known that $N(a) \equiv a^{p} \bmod p$ if $a \in \mathbf{Z}\left(\zeta_{2}\right)$, so we see that

$$
\begin{aligned}
k(z) & =\left(N f\left(\zeta_{2}\right)^{-1} f\left(\zeta_{1}\right)\right) \bmod p \equiv\left(f\left(\zeta_{2}\right)^{p}\right)^{-1} f\left(\zeta_{2}^{p}\right) \bmod p \\
& \equiv\left(f\left(\zeta_{2}\right)^{p}\right)^{-1} f\left(\zeta_{2}\right)^{p} \bmod p=1 .
\end{aligned}
$$

So $z \in \operatorname{Ker} k=\operatorname{Im} \lambda_{1} \oplus \lambda_{2}$. This implies there is an element $u \in R_{\pi}^{\times}$such that $\lambda_{1}(u)=\bar{u}, \lambda_{2}(u)=f\left(\zeta_{2}\right)$ and so $\operatorname{tr} u=N \lambda_{2}(u)=N f\left(\zeta_{2}\right)=\bar{u}$. Thus $\operatorname{tr}$ is onto and we are done.

Now we restate and prove

THEOREM 3. If $k$ is an odd integer, $n>1$, any free $\mathbf{Z} / k$ action on $S^{2 n+1}$ extends to a free $\mathbf{Z} / 2 k$ action.

Proof. According to Theorem 1 we have to prove that $\operatorname{tr}:\left(R_{\pi}\right)^{\times} \rightarrow\left(R_{\rho}\right)^{\times}$is onto, where $\pi=\mathbf{Z} / 2 k, \rho=\mathbf{Z} / k$. But there is an exact sequence of units (used earlier),

$$
1 \rightarrow(\mathbf{Z} \rho) \stackrel{i_{*}}{\rightarrow}\left(R_{\rho}\right) \stackrel{\times \varepsilon_{*}}{\rightarrow}(\mathbf{Z} / k)^{\times} /( \pm 1),
$$


where $\varepsilon_{*}$ is induced by the augmentation $\varepsilon: R \rho \rightarrow \mathbf{Z} / k$. Hence it is enough to prove: (a) $\operatorname{Im} i_{*} \subset \operatorname{Im} \operatorname{tr}$ where $i_{*}:(\mathbf{Z} \rho)^{\times} \rightarrow\left(R_{\rho}\right)^{\times}$is the map induced by projection and (b) $\varepsilon_{*} \circ \operatorname{tr}$ is onto.

The proof of (b) is elementary: Each unit of $(\mathbf{Z} / k)^{\times}$is of the form $2 r+1 \bmod k$ for some integer such that $0<2 r+1<2 k$. We claim that, in $(R \pi)^{\times}$the unit $u=\left(T^{2 r+1}-1\right) /(T-1)$ satisfies $\varepsilon_{*} \operatorname{tr} u=2 r+1$. For $u=1+T+\cdots+T^{2 r}=$ $\alpha+T^{k} \beta$ where $\alpha=1+T^{2}+\cdots+T^{2 r}, \beta=\left(T^{k+1}+T^{k+3}+\cdots+T^{k+2 r-1}\right)$. Note $\alpha$ and $\beta$ lie in the image of $\mathbf{Z} \rho$. Since $\pi=\rho \times \mathbf{Z} / 2$, it is an elementary exercise to see $\operatorname{tr} u=\alpha^{2}-\beta^{2} \bmod \bar{\Sigma}$. Set $\gamma=\alpha-T^{2 r}$, and note $\operatorname{tr} u=\left(\gamma+T^{2 r}\right)^{2}-$ $T^{2}\left(\gamma^{2}\right)=\left(1-T^{2}\right) \gamma^{2}+2 \gamma T^{2 r}+T^{4 r}$. Hence $\varepsilon_{*} \operatorname{tr} u=0 \cdot \varepsilon\left(\gamma^{2}\right)+2 \varepsilon(\gamma)+1=2 r$ +1 since $\varepsilon(\gamma)=r$. This proves $(\mathrm{b})$.

To prove (a) we observe that the diagram

$$
\begin{array}{ccc}
\mathbf{Z}(\pi)^{\times} & \rightarrow & (R \pi)^{\times} \\
\downarrow N & & \downarrow \text { tr } \\
\mathbf{Z}(\rho)^{\times} & \stackrel{i}{\rightarrow} & \left(R_{\rho}\right)^{\times}
\end{array}
$$

commutes, where $N$ is the norm map, so we can concentrate on proving that $N$ is onto. Now $\mathbf{Z} \pi \approx A(\mathbf{Z} / 2)$ where $A=\mathbf{Z}(\rho)$ so we show $N: A(\mathbf{Z} / 2)^{\times} \rightarrow A^{\times}$is onto. Each element of $A(\mathbf{Z} / 2)$ can be written $a+b S$, where $a, b \in A, S \in \mathbf{Z} / 2$. Also $N$ is given by $N(a+b S)=a^{2}-b^{2}$. An element $\nu=a+b S$ is a unit of $A(\mathbf{Z} / 2)$ if and only if $a+b=u$ and $a-b=u^{\prime}$ are units of $A$; conversely, given units $u, u^{\prime}$ in $A^{\times}$ with $u \equiv u^{\prime} \bmod 2 A$ they determine a unit $\nu$ uniquely. Note $N(\nu)=u u^{\prime}$.

Now, the diagram

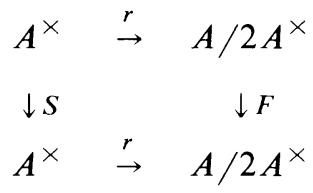

commutes where $r$ is reduction, $S(x)=x^{2}$ and $F$ is given by the Frobenius map $F(x)=x^{2} . F: A / 2 A \rightarrow A / 2 A$ is an isomorphism of rings because $A / 2 A$ is a product of finite fields of characteristic $2\left(A=\mathbf{Z}_{(\rho)}\right)$. It follows that $\operatorname{Im} r=\operatorname{Im} F \circ r$.

Now we prove $N: A(\mathbf{Z} / 2)^{\times} \rightarrow A^{\times}$is onto. Let $w \in A^{\times}$. By the last paragraph, $r(w)=r\left(u^{2}\right)$ for some $u \in A^{\times}$. Write $w$ as $w=u u^{\prime}$ for some $u^{\prime} \in A^{\times}$. Then $r\left(u^{\prime} / u\right)=r\left(w / u^{2}\right)=1$ so $u^{\prime} / u \equiv 1 \bmod 2 A$. It follows that $u \equiv u^{\prime} \bmod 2 A$ and so, as seen above, these determine a unit $\nu=\left(u+u^{\prime}\right) / 2+S\left(u-u^{\prime}\right) / 2$, in $A(\mathbf{Z} / 2)^{\times}$ with $N \nu=u u^{\prime}=w$ as required. This completes the proof.

\section{REFERENCES}

1. W. Browder, T. Petrie and C. T. C. Wall, The classification of free actions of cyclic groups of odd order on homotopy spheres, Bull. Amer. Math. Soc. 77 (1971), 455-459.

2. K. Iwasawa, On the theory of cyclotomic fields, Ann. of Math. 70 (1959), 530-561.

3. J. Milnor, Introduction to algebraic K-theory, Princeton Univ. Press, Princeton, N. J., 1971.

4. ,Whitehead torsion, Bull. Amer. Math. Soc. 72 (1966), 358-426.

5. T. Petrie, The Atiyah-Singer invariant, the Wall group $L_{n}(\pi, 1)$ and the function $\left(t e^{x}+1\right) /\left(t e^{x}-1\right)$, Ann. of Math. (2) 92 (1970), 174-187. 
6. D. Sullivan, Triangulating homotopy equivalences, Thesis, Princeton Univ., Princeton, N. J., 1966.

7. C. T. C. Wall, Classification of Hermitian forms. VI, Group rings, Ann. of Math. (2) 103 (1976), $1-80$.

8. __ Some L-groups of finite groups, Bull. Amer. Math. Soc. 79 (1973), 526-529.

9. __ Surgery on compact manifolds, Academic Press, New York, 1970.

10. H. Bass, Algebraic K-theory, Benjamin, New York, 1968.

11. J. Ewing, Extending free cyclic actions on spheres, Trans. Amer. Math. Soc. 273 (1982), 695-703.

12. S. Wagstaff, The irregular primes to 125,000, Math. Comp. 32 (1978), 583-591.

Department of Mathematics, University of Notre Dame, Notre Dame, Indiana 46556

Department of Computer Science, Duke University, Durham, North Carolina 27706 